\title{
ANALYTIC FORMULATION FOR 3D DIFFUSION TENSOR
}

\author{
C.PLATERO, G.ASENSION, P.GONZÁLEZ, \\ M.C.TOBAR,J.SANGUINO,J.M.PONCELA ${ }^{\dagger}$ \\ Applied Bioengineering Group, Polytechnic University of Madrid \\ c/ Ronda de Valencia 3 \\ Madrid, Spain
}

\begin{abstract}
This work deals with image processing based upon non-linear diffusion PDEs (Partial Differential Equations). Some analytic formulation will be introduced to obtain the 3D diffusion tensor, replacing Jacobi's numerical methods by expressions based on invariants of the symmetric matrix. Later, CED (Coherence Enhancing Filtering) anisotropic filtering properties will be observed and will be combined with isotropic diffusion, providing a type of filtering that allows combining noise removal and local structure preservation. Last, some applications 3D grey-level will be presented.
\end{abstract}

\section{Introduction}

For most applications making use of image enhancement, it is desirable to remove noise as much as possible and to increase the contrast, trying in both cases to preserve their structures. During the last decade, there has been great interest in the use of PDEs for image processing, mainly because they solve the disadvantage previously mentioned. There is the possibility to apply a certain filtering dependant on the characteristics of the area to be processed. Inside PDE there has been major focus on the non linear diffusion equation:

$$
\partial_{t} u=\operatorname{div}(g(|\nabla u|) \nabla u)
$$

Where $g(|\nabla u|)$ is the diffusivity, it is a function $g: \mathbb{R}^{m} \rightarrow \mathbb{R}$ ( $m$ is the dimension image), decreasing with the magnitude of the gradient module. There have been several proposals for this function during the last years [2]. Weickert [3] proposed a new diffusion framework, where diffusivity is not a scalar magnitude but a diffusion tensor:

$$
\partial_{t} u=\operatorname{div}(D \nabla u)
$$

\footnotetext{
${ }^{\dagger}$ Work supported by grant DPI-2007-63654 Spanish Ministry of Education and Sciences.
} 
where $D$ is an mxm semi-positive definite symmetric matrix. The $D$ tensor will be determined by the eigenvectors and eigenvalues of the specific tensor type:

$$
D=R C R^{T}
$$

$R$ is the rotation matrix and $C$ is the diagonal matrix, whose elements correspond to the diffusion coefficients in each of the main components. The diffusion process is based on the gradient is projected onto the tensor. The smooth effect is increasing in the directions with less variability.

\section{Anisotropic diffusion of the local coherence enhancement}

Different tensor types will be defined depending on the specific application. For example, for applications in which it is important to recover the local coherence of the image structure - generally altered by acquisition systems - Weickert [3] proposed to use a local coherence enhancement diffusion tensor (CED Coherence Enhancing Filtering). The tensor structure is based on the product of the gradient descriptor smoothed with its transposed and convolutioned with the Gaussian mask $K_{\rho}$ :

$$
S=K_{\rho} *\left(\nabla u_{\sigma} \nabla u_{\sigma}^{T}\right) \quad \nabla u_{\sigma}=\nabla\left(K_{\sigma}^{*} u\right) \quad K_{\sigma}=\frac{1}{\left(2 \pi \sigma^{2}\right)^{m / 2}} \exp \left(-\frac{|x|^{2}}{2 \sigma^{2}}\right) \text { (4) }
$$

Matrix $S$ is invariant under changes in the sign of the gradient direction, but it is dependant on its magnitude. The value for the standard deviation depends on the noise scale $\sigma$ and the value for $\rho$ depends on the local orientation. The eigenvector of matrix $S$, for $\rho=0$, are parallel and orthogonal to $\nabla u_{\sigma}$. After the last convolution - with $\rho$ - there has been a change from the gradient specific direction to the local structure main orientation. $S$ is known as structure tensor or second moment matrix.

To enhance local patterns it is proposed that the coefficients of the diagonal matrix $C$ are given by:

$$
c_{1}=\left\{\begin{array}{cc}
\alpha & k=0 \\
\alpha+(1-\alpha) \exp \left(-\frac{\beta}{\kappa}\right) & k \neq 0
\end{array} \quad c_{i}=\alpha \quad i=2, . ., m\right.
$$


Having sorted out the components from minor to major eigenvalues. Therefore, the first component will show the direction of highest local homogeneity in the irradiation level, that is, of lowest variability in the direction of this main component. The value for $\beta$ is a threshold that is compared to the local coherence level, $\kappa$, and $\alpha$ is a control parameter whose value is within the interval [0-1], to guarantee the diffusion tensor smoothing [3]. In order to measure whether a neighborhood boundary is homogeneous or not, the local coherence measure is introduced:

$$
\kappa=\sum_{i=1}^{m} \sum_{i \neq j}\left(\mu_{i}-\mu_{j}\right)^{2}
$$

where $\mu_{i}$ is the eigenvector of the main component $i$ in $S($ ). If eigenvalues are similar, the coherence level $-\kappa$ - will be low and the diffusion coefficients will be similar, in which case there will be an almost isotropic diffusion process. When differences among the eigenvalues appear, there will be a boundary with main components highlighted with respect to the others, creating an anisotropic diffusion process in the direction of minimum variability.

For 2D images, the CED tensor analytical expressions are extended [4]. However, in the case of 3D images, numerical methods are generally used, implementing multiple variations of Jacobi's numerical methods. But as an alternative, exact solutions can be found through the invariants mathematical theory (scalar functions of the matrix that do not vary after a base change, just as the determinant). The calculation of the eigenvalues of a $3^{\text {rd }}$ order symmetric tensor requires the introduction of the following invariants of the second moment matrix:

$$
I_{1}=\operatorname{tr}(S) \quad I_{2}=\frac{1}{2}\left(\operatorname{tr}\left(S^{2}\right)-\operatorname{tr}(S)^{2}\right) \quad I_{3}=\operatorname{det}(S)
$$

which are used to calculate the invariants of the deflector component of matrix $S$, $\tilde{S}=S-\frac{1}{3} I_{1} I$ :

$$
J_{2}=\frac{1}{2}\left(\operatorname{tr}\left(\tilde{S}^{2}\right)-\operatorname{tr}(\tilde{S})^{2}\right)=\frac{1}{3}\left(I_{1}^{2}+3 I_{2}\right) \quad J_{3}=\operatorname{det}(\tilde{S})=\frac{1}{27}\left(2 I_{1}^{3}+9 I_{1} I_{2}+27 I_{3}\right)(8)
$$

which afterwards allows calculating Lode's angle:

$$
\cos (3 \theta)=\frac{3 \sqrt{3}}{2} \frac{J_{3}}{J_{2}^{3 / 2}} \quad 0 \leq \theta \leq \frac{\pi}{3}
$$


And it can be demonstrated (Asensio and Moreno [1]) that the eigenvalues of tensor $S$ - sorted out from minor to major - are:

$$
\mu_{1,2}=\frac{1}{3} I_{1}+2 \sqrt{\frac{J_{2}}{3}} \cos \left(\theta \pm \frac{2 \pi}{3}\right) \quad \mu_{3}=\frac{1}{3} I_{1}+2 \sqrt{\frac{J_{2}}{3}} \cos (\theta)
$$

The calculation of the tensor elements will be a function of the second moment matrix, of the diffusivity coefficients in each component and of the eigenvalues. The diffusion tensor $D$ is rebuilt from the rotation matrix $R$ and from a diagonal diffusion matrix $C$, according to $C=R D R^{t}$, therefore:

$$
D_{i j}=c_{1} R_{1 i} R_{1 j}+c_{2} R_{2 i} R_{2 j}+c_{3} R_{3 i} R_{3 j}
$$

obtaining the following:

$$
\begin{aligned}
& D_{i j}=\sum_{k=1}^{k} \frac{c_{k}\left(\tilde{S}_{i 3}+s_{i 3} \mu_{k}\right)\left(\tilde{S}_{j 3}+s_{j 3} \mu_{k}\right)}{\left(\tilde{S}_{i 3}+S_{j 3} \mu_{k}\right)^{2}+\left(\tilde{S}_{j 3}+S_{j 3} \mu_{k}\right)^{2}+\left(\tilde{S}_{33}+\left(s_{11}+s_{22}\right) \mu_{k}-\mu_{k}^{2}\right)^{2}} \text { if } i, j=1,2 \\
& D_{i 3}=\sum_{k=1}^{k} \frac{c_{k}\left(\tilde{S}_{i 3}+S_{i 3} \mu_{k}\right)\left(\tilde{S}_{33}+\left(s_{11}+s_{22}\right) \mu_{k}-\mu_{k}^{2}\right)}{\left(\tilde{S}_{i 3}+s_{j 3} \mu_{k}\right)^{2}+\left(\tilde{S}_{j 3}+s_{j 3} \mu_{k}\right)^{2}+\left(\tilde{S}_{33}+\left(s_{11}+s_{22}\right) \mu_{k}-\mu_{k}^{2}\right)^{2}} \text { if } i=1,2 \\
& D_{33}=\sum_{k=1}^{k} \frac{c_{k}\left(\tilde{S}_{33}+\left(s_{11}+S_{22}\right) \mu_{k}-\mu_{k}^{2}\right)^{2}}{\left(\tilde{S}_{i 3}+s_{j 3} \mu_{k}\right)^{2}+\left(\tilde{S}_{j 3}+s_{j 3} \mu_{k}\right)^{2}+\left(\tilde{S}_{33}+\left(s_{11}+s_{22}\right) \mu_{k}-\mu_{k}^{2}\right)^{2}}
\end{aligned}
$$

\section{CED isotropic and anisotropic diffusion conditions}

For flat areas, whose gray level is almost constant, the eigenvalues of the second moment matrix are very similar and low values. Consequently, all the components will have diffusivity coefficients with very close values. In this case the tensor becomes a diagonal matrix weighted by the diffusion parameter $\alpha$, and there is isotropic diffusion. On the other hand, when the eigenvalues of the structure tensor are very different, there is anisotropic diffusion and it is predominant along the direction perpendicular to the gradient. The decision for a given pixel to perform isotropic or anisotropic diffusion depends on the threshold level $\beta$ and the local coherence measure, $\kappa$. According to equation 5, there will be anisotropic diffusion when the local coherence measure is greater than zero, but if the condition is loosened, it will be possible to define the threshold level $\beta$ considering the image information. Thus, once the local coherence measure $\kappa$ is obtained, its dynamic range will be known, and it will be possible to define the value for $\beta$ : 


$$
\beta=-\kappa^{*} \cdot \log \left(\frac{\alpha}{f} \frac{1}{1-\alpha}\right)
$$

Where $f$ is the fraction of $\alpha$ considered significant to move from isotropic to anisotropic diffusion, and $\kappa^{*}$ is the local coherence measure level at which anisotropic diffusion will start to be applied. Therefore, for local coherence measure greater than $\kappa^{*}$ there will be anisotropic diffusion, and in the opposite case there will be isotropic diffusion. For example, if the anisotropic diffusion process is to begin at a coherence index such that for $\alpha=0.01$ it is doubled, $f=$ 1 , the diffusion tensor will have to be calculated for local coherence measure starting from a fifth of $\beta$. Thus, it will be possible to define the threshold value $\beta$ thanks to a previous view of the coherence index for each individual pixel.

Besides, this property can be used to spare computation time due to the fact that if the local coherence measure $\kappa$ is smaller than $\kappa^{*}$, the tensor will not be calculated and will be substituted by the diagonal matrix $I \alpha$. In the opposite case, the tensor will be calculated. The local coherence measure for 3D images correspondence to:

$$
\kappa=\sum_{i=1}^{3} \sum_{i \neq j}\left(\mu_{i}-\mu_{j}\right)^{2}=6 J_{2}
$$

When this property is used, the reduction in computation times will depend on the nature of the processed images.

\section{Combination of isotropic and anisotropic diffusion}

Figures 1 show a 3D diffusion application to reconstruction a mother neuron using confocal microscopy. The source shows some noise in the acquisition stage. Isotropic diffusion shows good results in removing noise, but it does not succeed to preserve the structure of the biological object. When CED is applied some fake dendrites appear due to the enhancement of patterns outside the vision field. The best results are obtained when isotropic and anisotropic diffusion is combined. 


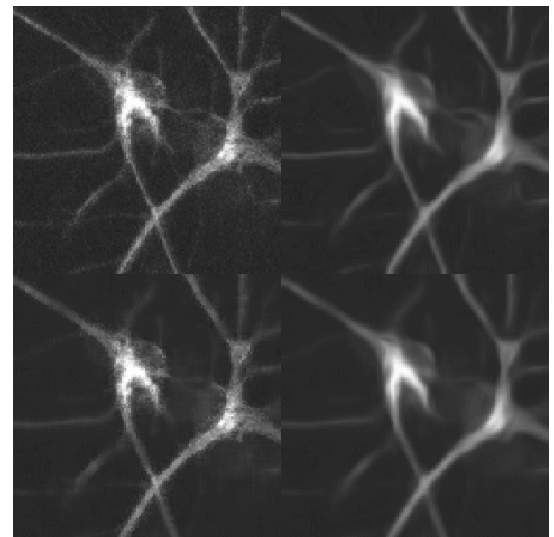

Figure 1 a) Slice number 34 from a mother neuron image collection obtained through confocal microscopy b) Image processed through CED c) Image processed through isotropic diffusion d) Image processed through isotropic diffusion and CED diffusion.

\section{Conclusions}

We have presented here the theoretical framework for isotropic and anisotropic diffusion, paying special attention to CED anisotropic diffusion. Three aspects have been studied: 3D tensor analytical expressions, CED parameters and improvements of isotropic and anisotropic diffusion combination.

\section{References}

1. M G. Asensio, C. Moreno, Linearization and return mapping Algorithms for elastoplasticity models, International Journal for Numerical Methods in Engineering, 57, 991-1014, (2003).

2. G. Aubert, P. Kornprobst, Mathematical Problems in Image Processing, Applied Mathematical Sciences 147, Springer-Verlag New-York, (2006).

3. J. Weickert, Coherence-enhancing diffusion filtering, International Journal of Computer Vision, 31, 111-127, (1999).

4. J. Weickert, H. Scharr, A scheme for coherence-enhancing diffusion filtering with optimized rotation invariance, Journal of Visual Communication and Image Representation, 13(1), 103-118, (2002). 